\title{
Vector potential gauge for superconducting regular polygons
}

\author{
L.F. Chibotaru ${ }^{1, a}$, A. Ceulemans ${ }^{1}$, G. Teniers ${ }^{2}$, V. Bruyndoncx ${ }^{2}$, and V.V. Moshchalkov² \\ 1 Katholieke Universiteit Leuven, Afdeling Kwantumchemie, Celestijnenlaan 200F, 3001 Leuven, Belgium \\ 2 Katholieke Universiteit Leuven, Laboratorium voor Vaste-Stoffysica en Magnetisme, Celestijnenlaan 200D, \\ 3001 Leuven, Belgium
}

Received 28 February 2002 and Received in final form 12 April 2002

Published online 6 June 2002 - (c) EDP Sciences, Società Italiana di Fisica, Springer-Verlag 2002

\begin{abstract}
An approach to the Ginzburg-Landau problem of superconducting polygons is developed, based on the exact fulfillment of superconducting boundary conditions along the boundary of the sample. To this end an analytical gauge transformation for the vector potential $\mathbf{A}$ is found which gives $A_{n}=0$ for the normal component along the boundary line of an arbitrary regular polygon. The use of the new gauge reduces the Ginzburg-Landau problem of superconducting polygons in external magnetic fields to an eigenvalue problem in a basis set of functions obeying Neumann boundary conditions. The advantages of this approach, especially for low magnetic fields, are illustrated and novel vortex patterns are obtained which can be probed experimentally.
\end{abstract}

PACS. 74.60.Ec Mixed state, critical fields, and surface sheath - 74.25.Dw Superconductivity phase diagrams - 74.20.De Phenomenological theories (two-fluid, Ginzburg-Landau, etc.)

\section{Introduction}

Recent progress in microfabrication techniques makes it possible to investigate mesoscopic superconducting samples with sizes smaller than the coherence length and the penetration depth [1]. The new qualitative feature arising at the mesoscopic scale is the strong effect that the boundary geometry has on the nucleation of superconductivity in the samples. The theoretical description of the superconductivity in applied magnetic field requires the solution of the Ginzburg-Landau equations [2] with the boundary conditions imposed on the superconducting order parameter $\psi$ at the superconductor/vacuum interface:

$$
\left.\left(-\mathrm{i} \hbar \nabla-\frac{2 e}{c} \mathbf{A}\right) \psi\right|_{n}=0,
$$

where $\mathbf{A}$ is the vector potential corresponding to the magnetic field.

The presence of the vector potential in the boundary condition, equation (1), seriously complicates the solution of the Ginzburg-Landau equations for samples of arbitrary geometry. Existing treatments use numerical methods like the method of finite differences $[3,4]$. However the problem can be viewed from a different perspective if one can find a gauge for the vector potential which gives $A_{n}=0$ for the normal component along the boundary line. In these cases the superconducting boundary condition in equation (1)

\footnotetext{
a e-mail: liviu.chibotaru@chem.kuleuven.ac.be
}

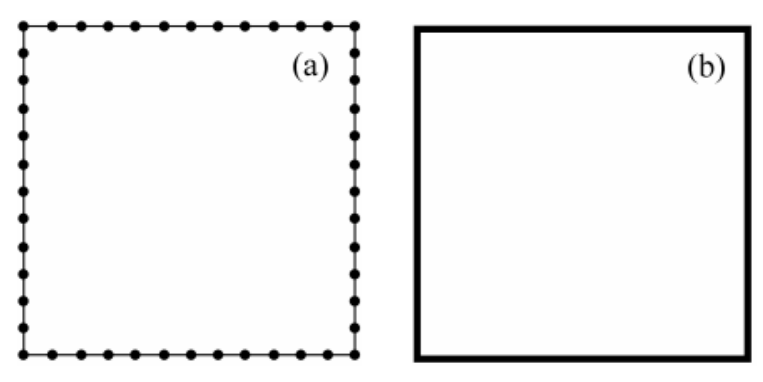

Fig. 1. Domains (bold dots and lines) of the boundary line where the condition in equation (1) is satisfied exactly for the case of finite grid (a) and appropriate vector potential gauge (b) methods.

reduces to the Neumann boundary condition, $\left.\nabla \psi\right|_{n}=0$, which is much easier to satisfy. Such a choice of the gauge has been achieved till now only for infinite slabs [5], semiplanes with a wedge $[6,7]$, and disks [8-11].

The major difference between the above approaches is the extent to which they fulfil the superconducting boundary condition. As Figure 1 shows, the method of finite differences satisfies equation (1) only on a finite set of points on the boundary line, while by using an appropriate gauge for the vector potential we are able to satisfy the boundary condition everywhere on the boundary. Since equation (1) is nothing but a sort of quantization condition for our problem, we can conclude that finite grid 
methods always imply an approximate solution of equation (1), while the methods using the appropriate gauge for the vector potential treat it exactly. This is crucial for the proper description of the order parameter in the cases when the boundary becomes important, i.e. for low values of the applied flux.

In this paper we develop this second type of approach to the Ginzburg-Landau problem for regular superconducting polygons in external magnetic field. For these geometries, an analytical gauge transformation for the vector potential of a homogeneous magnetic field yielding $A_{n}=0$ on the boundary line of arbitrary regular polygons is found. Vector potential is assumed to originate uniquely from the applied field. This approach is valid in the cases when the screening effect of the induced supercurrents can be neglected. In macroscopic superconductors this criterion is only fulfilled close to the phase boundary for the nucleation of superconductivity, while in mesoscopic superconductors it is satisfied in a wide range of temperatures and fields if the samples are thin enough [12]. The advantages of the developed approach are illustrated for the solution of the linearized Ginzburg-Landau equation in a square. It has already been successfully applied to the description of the nucleation of superconductivity in mesoscopic squares and triangles $[13,14]$ which are started to be fabricated and investigated experimentally [15].

\section{General approach}

Consider a regular polygon with $N$ edges. It has a symmetry axis of order $N$, corresponding to rotations by angles which are multiples of $2 \pi / N$. An external homogeneous magnetic field applied along this axis can be described by a vector potential

$$
\mathbf{A}=\frac{1}{2} \mathbf{H} \times \mathbf{r}
$$

where the radius-vector lies in the $x y$ plane (Fig. 2) and $\mathbf{H} \| \mathbf{z}$. Equation (2) defines the cylindrical gauge for $\mathbf{A}$. It is often preferred over many other possible choices [16] due to the high symmetry which allows to preserve the rotational symmetry of the system without field. The direction of $\mathbf{A}$ is tangential to concentric circumferences, A $\| \mathbf{e}_{\varphi}$, which are also equipotential lines for the vector potential.

As one can see from Figure 2, the vector potential in equation (2) is not tangential to the boundary line (i.e. the edges) of a polygon. On the edge shown in Figure 2 it contains a normal component

$$
\begin{aligned}
A_{n}(\varphi) & =-C \tan \varphi, \\
C & =\frac{1}{4} H a,
\end{aligned}
$$

where $\varphi$ is the polar angle and $a$ is the diameter of the circumference inscribed in the regular polygon. Our purpose is to find a new vector potential which would be tangential to the edges. This can be done by the following gauge

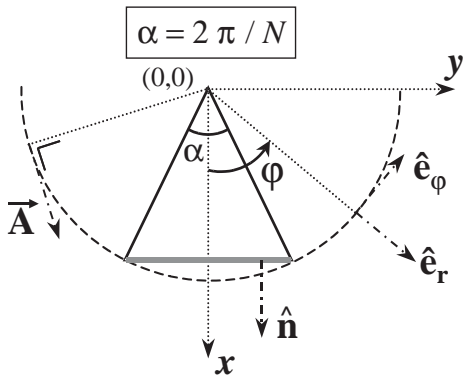

Fig. 2. Piece of a regular polygon containing one edge (thick line). The origin of the coordinate system is chosen in the centre of the polygon. $\mathbf{n}$ is the unit vector normal to the edge. $\mathbf{e}_{\mathbf{r}}$ and $\mathbf{e}_{\varphi}$ are unit vectors of the cylindrical coordinate system. The $z$ axis coincides with the $N$ fold rotational axis of the polygon.

transformation [16]:

$$
\tilde{\mathbf{A}}=\mathbf{A}+\nabla S
$$

where $\mathbf{A}$ is the old and $\tilde{\mathbf{A}}$ is the new vector potential and $S$ is an arbitrary scalar function. This function is found from the condition $\tilde{A}_{n}=0$ on the boundary line. This is equivalent to the equation

$$
A_{n}=-\nabla_{n} S,
$$

which has to be obeyed on each edge. In order to keep the rotational symmetry of the regular polygon $\tilde{\mathbf{A}}$ and $S$ should be periodic functions of $\varphi$ with the period $\alpha$ (Fig. 2). Because $\tilde{\mathbf{A}}$ is real, this suggests the following general form for $S$ (in polar coordinates):

$$
S(r, \varphi)=\sum_{m}\left[R_{m}^{s}(r) \sin (N m \varphi)+R_{m}^{c}(r) \cos (N m \varphi)\right]
$$

where $m$ are non-negative integers. Due to the rotational periodicity of the function $S$ it is enough to satisfy equation (5) on one single edge. The form in equation (6) is further simplified due to the symmetry requirement that $\tilde{\mathbf{A}}$ is purely tangential on the radial lines defined by $\varphi= \pm \alpha / 2$ and $\varphi=0$ (Fig. 2). The latter requirement means that $\nabla S$ is purely tangential on the radial line $\varphi=0$ which can only be the case if one takes $R_{m}^{c}(r)=0$ in equation (6).

Next we simplify the remaining part of the form (6) by confining ourselves to one single term in the summation. Obviously this cannot be the term $m=0$ because $\nabla S$ should be dependent on $\varphi$ as it is easily seen from equations $(3,5)$. Therefore the simplest possible term is $m=1$ which leads to the ansatz:

$$
S_{N}(r, \varphi)=R_{N}(r) \sin (N \varphi) .
$$

Substituting equations $(7,3)$ into $(5)$, after eliminating the $r$ variable on the edge line:

$$
\begin{aligned}
r & =\frac{a}{2 \cos \varphi}, \\
\frac{\partial}{\partial r} & =\frac{2}{a} \frac{\cos ^{2} \varphi}{\sin \varphi} \frac{\partial}{\partial \varphi}
\end{aligned}
$$


one obtains the following equation in $\varphi$ :

$$
\tilde{R}_{N}^{\prime} \sin (N \varphi) \cos ^{2} \varphi-\tilde{R}_{N} \sin (N \varphi)^{\prime} \sin ^{2} \varphi=\tan ^{2} \varphi
$$

where the prime in the superscript means the first derivative after $\varphi$ and the following notation was introduced:

$$
\tilde{R}_{N}(\varphi)=\frac{2}{a C} R_{N}\left(\frac{a}{2 \cos \varphi}\right)
$$

Bringing equation (9) to the form

$$
\tilde{R}_{N}^{\prime}+\xi(\varphi) \tilde{R}_{N}=\eta(\varphi)
$$

where

$$
\begin{aligned}
\xi(\varphi) & =-N \cot (N \varphi) \tan ^{2} \varphi, \\
\eta(\varphi) & =\csc (N \varphi) \tan ^{2} \varphi / \cos ^{2} \varphi,
\end{aligned}
$$

allows us to write down the general solution [17]:

$$
\begin{aligned}
\tilde{R}_{N}(\varphi) & =\frac{1}{\mu(\varphi)}\left[\int \eta(\varphi) \mu(\varphi) \mathrm{d} \varphi+C_{1}\right], \\
\mu(\varphi) & =\exp \left[\int \xi(\varphi) \mathrm{d} \varphi\right] .
\end{aligned}
$$

The solution (13) describes the radial function in equation (7) only for values of $r$ which are radius vectors of the points on the edge's line. One can extend this solution over the whole range of $r$ by the inverse transformation to $(8), a / 2 \cos \varphi \rightarrow r$. Then using again equations $(10,7)$ and (4) we can express the polar components of the gauge transformed vector potential $\tilde{\mathbf{A}}$,

$$
\begin{aligned}
& \tilde{A}_{r}=\frac{\partial R_{N}(r)}{\partial r} \sin (N \varphi), \\
& \tilde{A}_{\varphi}=\frac{1}{2} H r+\frac{N}{r} R_{N}(r) \cos (N \varphi),
\end{aligned}
$$

through the solution (13). The constant $C_{1}$ contained in that solution should be chosen in such a way as to provide nondivergent components of the vector potential (14). In contrast to $\mathbf{A}$ in equation (2), the vector potential defined by equations (14) does not obey the Coulomb gauge, $\nabla \cdot \mathbf{A}=0$. Hence the term $\nabla \cdot \mathbf{A}$ plays the role of a scalar potential in an Hamiltonian and should be nondivergent either. Fortunately both requirements are met within the area of the polygon under the simple condition $C_{1}=0$.

The general solutions (13) for polygons with odd and even vertices are further specified in the Appendix, together with analytical expressions for some particular cases of small $N$ 's.

\section{Application to the Ginzburg-Landau problem}

The analytical results for the gauge transformed vector potentials can be used directly in the Ginzburg-Landau

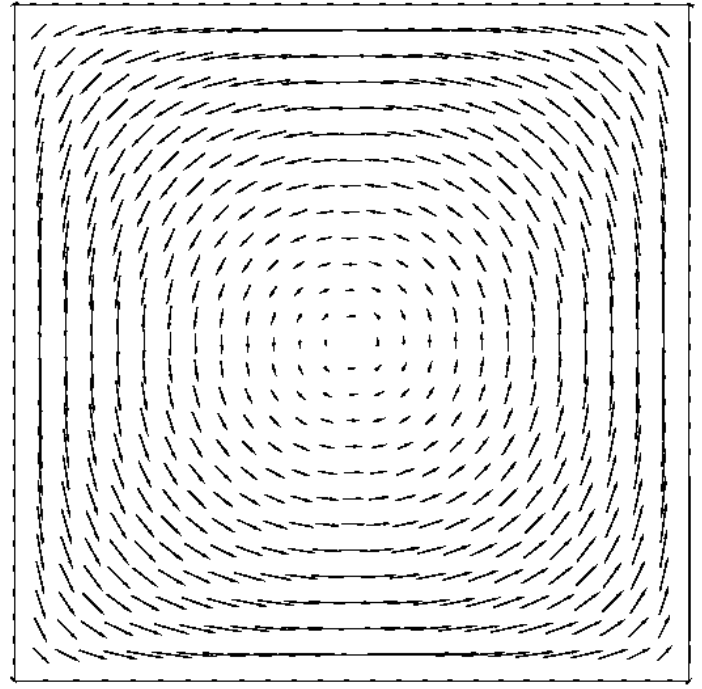

Fig. 3. Vector potential for the square (arbitrary units) after the gauge transformation, described by equations (A.13).

equations [2] describing regular superconducting polygons. In the case of square geometry the components $\tilde{A}_{r}$ and $\tilde{A}_{\varphi}$ are given by the formulae (A.13), and Figure 3 shows an arrow representation of the resulting vector potential. As can be seen from this figure the vector potential in the central region has approximate cylindrical symmetry, corresponding to $\mathbf{A}$ in equation (2). It smoothly changes into a square symmetry pattern when approaching the boundary. Although $\tilde{\mathbf{A}}$ becomes tangential to the edges, they do not correspond to equipotential lines. Actually the vector potential reaches its maximal value in the middle of the edges $(|\tilde{\mathbf{A}}|=H a / 2)$ and is minimal in the corners $(\tilde{\mathbf{A}}=0)$. These features are common to other polygons as well.

The solutions for the square can be classified after irreducible representations (irreps) $A, B, E_{+}$and $E_{-}$of the symmetry group $C_{4}$ of the problem [18]. It turned out that a convenient basis set for the calculations is provided by eigenfunctions of the particle in a square box problem obeying Neumann boundary conditions [13]. For each irrep, the Hamiltonian matrix is set up within a restricted basis set of these functions, of the given symmetry and corresponding to the lowest energy levels of the zero-field problem. The resulting lowest "eigenvalue" describes the $T-H$ phase boundary for the nucleation of superconductivity, while the lowest "eigenfunction", $\Psi$, corresponds to the order parameter at this phase boundary.

As an example, we further analyse the solution of the linearised Ginsburg-Landau equation for a superconducting square in an applied magnetic field generating the total flux $\Phi=5.5 \Phi_{0}$, where $\Phi_{0}$ is the superconducting flux quantum [2]. The stable region on the phase diagram corresponds to vorticity $L=3, E_{-}$symmetry of the order parameter, and the vortex structure [19] is characterized by one $\Phi_{0}$-antivortex in the centre and four $\Phi_{0^{-}}$ vortices symmetrically dispatched along the diagonals of 

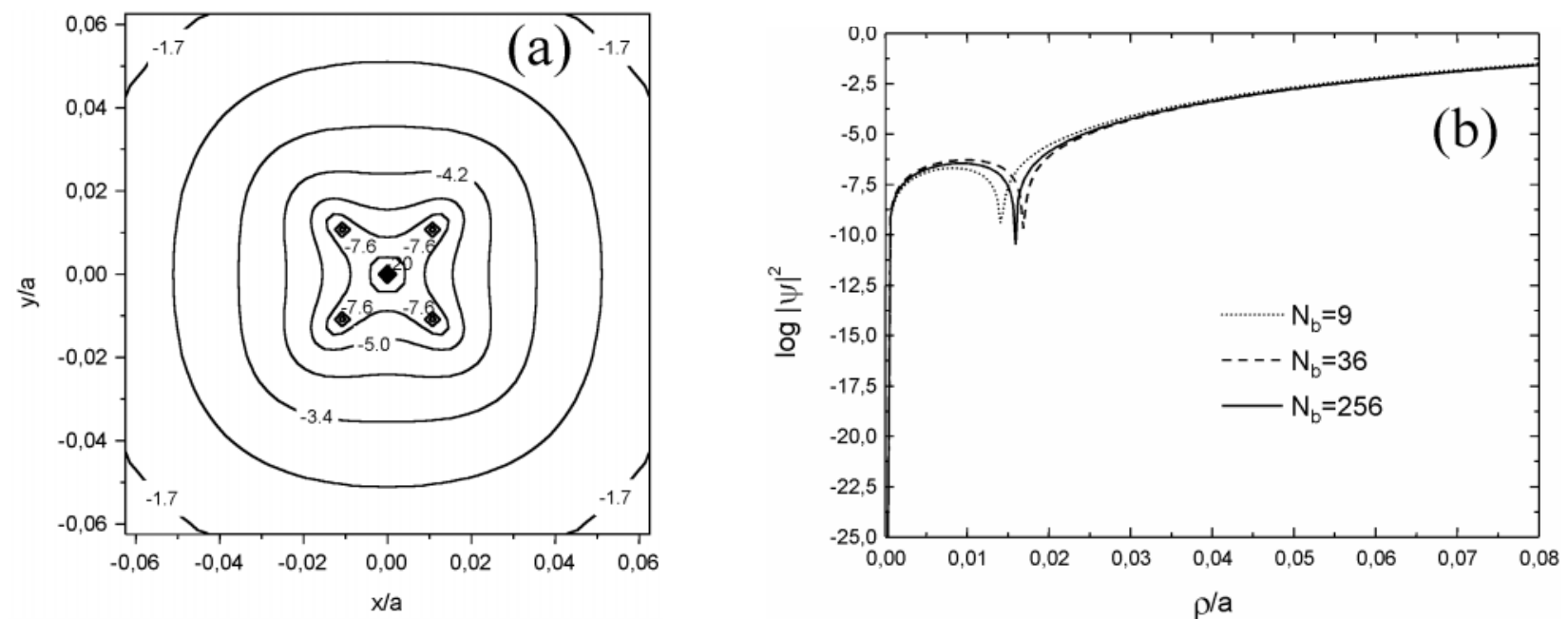

Fig. 4. Order parameter plots corresponding to the solution of linearized Ginzburg-Landau equation for a square with gauge transformed vector potential as in equations (A.13) (the order parameter is normalized to unity). The contour plot (a) shows (in logarithmic scale) the vortex pattern in the central region of the square zoomed in eight times after convergence with respect to the basis set size was achieved. The pannel (b) displays the cross section in the diagonal direction ( $\rho=0$ is the centre of the square) for different numbers of basis basis functions used in the calculations: $N_{b}=9$ (dotted), 36 (dashed) and 256 (solid) the value at which convergence was achieved.

the square [13]. As Figure 4 shows, these "diagonal" vortices are shifted from the central position by an amount smaller than 2 percents of the square edge. If one uses a numerical technique with a lower resolution, the four vortices and the single antivortex would appear as one $3 \Phi_{0}$-giant vortex. Interestingly, the fine details of the order parameter structure are well reproduced already in a quite small basis set comprising the nine lowest functions of symmetry $E_{-}$. Indeed, it follows from Figure $4 \mathrm{~b}$ that the positions of the "diagonal" vortices resulting from the calculation with nine basis functions differ by only 12 percents from the "exact" values obtained in a calculation with 256 basis functions which reached the convergence after the basis set size.

Though the problem of measurability of the separate vortices in the obtained vortex pattern will be given due consideration elsewhere [20], we stress here that these vortices are not an artifact of the calculation. This is confirmed by the fast convergence of the solution with increasing basis set (Fig. 4b). The predicted novel vortex patterns (as, for example, in Fig. 4a) are found in the framework of the linearized Ginzburg-Landau equation, which is valid only quite close to the $T_{c}(H)$ phase boundary. In our calculations we have also used the local magnetic fields $\mathbf{b}(\mathbf{r})$ coinciding with the applied field. Therefore for the experimental verification of the new symmetry consistent vortex patterns close to $T_{c}(H)$, one should use such techniques which are sensible to the superfluid density, i.e. to $|\Psi|^{2}$, rather than to the variations in $\mathbf{b}(\mathbf{r})$. The former can be probed, for example, by using scanning tunneling microscopy.

It is worthwhile to compare the results in Figure 4 with those given by the finite differences method in the same region of field. In such calculations, the minima of the order parameter in the directions of the diagonals of the square only start to show up at a density of grid points not less than $201 \times 201$ [21]. For such a density of the grid, the matrix of the discretized Hamiltonian of dimension 40401 has to be diagonalized.

\section{Conclusions}

We have developed an approach to describe the superconductivity of mesoscopic regular polygons in homogeneous magnetic field based on the exact fulfillment of the superconducting boundary condition. To this end an analytical gauge transformation for the vector potential was derived which gives a vanishing normal component of the vector potential on the boundary line of any regular polygon. This transformation eliminates the vector potential from the boundary condition in equation (1). As a result the corresponding Ginzburg-Landau problem reduces to an eigenvalue equation in a basis set of functions obeying Neumann boundary conditions, which can be found for different regular polygons. The resulting slowly varying vector potential which is of the same order of magnitude as the initial, cylindrical gauge, allows one to use small basis sets for this eigenvalue problem as we have demonstrated for the solutions of the linear Ginzburg-Landau equations in a square (Fig. 4). In the field regions corresponding to several flux quanta, a satisfactory solution for the order parameter is obtained already with a basis set of 10 functions.

The main area of application of the above approach is expected to be the description of superconductivity in polygon-shaped mesoscopic samples in applied homogeneous magnetic fields. This is an important issue for physics of Josephson junctions [23] where different superconducting elements with a characteristic size in the range 
of $1-10 \mu \mathrm{m}$ are used. The achieved continuous description of the order parameter permits to calculate correctly the spatial changes of its phase gradient and to treat any regions of a superconductor no matter how small they are, and irrespective of the level of accuracy of the calculations. As a result new vortex patterns could be found in superconducting regular polygons displaying an antivortex in the centre of the polygon for some values of applied flux $[13,14]$. These can be probed by scanning tunneling microscopy.

The proposed vector potential gauge can also be used for the calculation of the magnetic response of type-II superconductors in realistic geometries [24] under the influence of the edge barrier.

Besides the superconductor/vacuum boundary conditions considered here, our approach can also be generalized to superconductor/metal-like boundaries.

We are grateful to V.V. Kabanov and J. Bonca for sharing with us the results of their calculations. This work is supported by the Flemish FWO, GOA, the Belgian IUAP and the ESF VORTEX Programmes.

\section{Appendix}

For the cases of small $N$ we derive explicit expressions for (14). To this end the trigonometric functions of $N \varphi$ in the definition of $\xi$ and $\eta$ in (12) are decomposed in powers of trigonometric functions of $\varphi$. With these decompositions, equation (11) can be shown to depend only on $\cos \varphi$ for any $N$. The further procedure differs for even and odd $N$.

\section{A.1 Odd N solutions}

Introducing the new variable

$$
v=\cos \varphi
$$

transforms equations $(11,12)$ into

$$
\frac{\partial \tilde{R}_{N}}{\partial v}+\xi_{o}(v) \tilde{R}_{N}=\eta_{o}(v)
$$

and

$$
\begin{aligned}
& \xi_{o}(v)= \\
& \frac{N}{v} \frac{\sum_{l=0}^{(N-1) / 2}(-1)^{l}\left(\begin{array}{c}
N \\
2 l
\end{array}\right) \sum_{k=0}^{l}(-1)^{k}\left(\begin{array}{l}
l \\
k
\end{array}\right) v^{N-2 l+2 k-1}}{\sum_{l=0}^{(N-1) / 2}(-1)^{l}\left(\begin{array}{c}
N \\
2 l+1
\end{array}\right) \sum_{k=0}^{l}(-1)^{k}\left(\begin{array}{l}
l \\
k
\end{array}\right) v^{N-2 l+2 k-1}}, \\
& \eta_{o}(v)=-\frac{1}{v^{4}} \\
& \quad \times \frac{1}{\sum_{l=0}^{(N-1) / 2}(-1)^{l}\left(\begin{array}{c}
N \\
2 l+1
\end{array}\right) \sum_{k=0}^{l}(-1)^{k}\left(\begin{array}{l}
l \\
k
\end{array}\right) v^{N-2 l+2 k-1}} .
\end{aligned}
$$

In this way the functions $\xi_{0}$ and $\eta_{o}$ become ratios of polynomials of order $N-1$. The solutions of equation (A.2) are similar to (13):

$$
\begin{aligned}
\tilde{R}_{N}(v) & =\frac{1}{\mu(v)} \int \eta_{o}(v) \mu(v) \mathrm{d} v, \\
\mu(v) & =\exp \left[\int \xi_{o}(v) \mathrm{d} v\right] .
\end{aligned}
$$

\section{A.1.1 Equilateral triangle}

Substituting $N=3$ in equations (A.3) gives the following expressions for the solution (A.4):

$$
\begin{aligned}
\mu(v) & =\frac{v^{9}}{\left(-1+4 v^{2}\right)^{3}} \\
\tilde{R}_{3}(v) & =\frac{1-12 v^{2}+48 v^{4}}{384 v^{9}} .
\end{aligned}
$$

Passing to $r$ in the last equation via the transformation $v \rightarrow a / 2 r$ one obtains for the components of $\tilde{\mathbf{A}}$ in (14):

$$
\begin{aligned}
\tilde{A}_{r} & =\frac{27}{2} H a\left(9 r^{\prime 8}-7 r^{\prime 6}+\frac{5}{3} r^{\prime 4}\right) \sin 3 \varphi \\
\tilde{A}_{\varphi} & =\frac{1}{2} H r+\frac{81}{2} H a\left({r^{\prime}}^{8}-r^{\prime 6}+\frac{1}{3} r^{\prime 4}\right) \cos 3 \varphi \\
r^{\prime} & =r / \sqrt{3} a .
\end{aligned}
$$

\section{A.1.2 Regular pentagon}

In the case $N=5$ the polynomials in r.h.s. of equations (A.3) are of the fourth order. The solution for $\tilde{R}_{5}$ is obtained in the form of one single integral:

$$
\begin{aligned}
\mu(v)= & \frac{\left(3+\sqrt{5}-8 v^{2}\right)^{\frac{1}{4}(30-17 \sqrt{5})}}{v^{25}} \\
& \times\left(-3+\sqrt{5}+8 v^{2}\right)^{\frac{1}{4}(30+17 \sqrt{5})}, \\
\tilde{R}_{5}(v)= & -\frac{1}{\mu(v)} \int \frac{\mu(v)}{v^{4}\left(16 v^{4}-12 v^{2}+1\right)} \mathrm{d} v .
\end{aligned}
$$

\section{A.2 Even $\mathrm{N}$ solutions}

In the case of even $N$ 's the expressions in equation (A.3) can further be simplified. Indeed, introducing the variable

$$
u=1 / \cos ^{2} \varphi
$$

transforms equations $(11,12)$ into

$$
\frac{\partial \tilde{R}_{N}}{\partial u}+\xi_{e}(u) \tilde{R}_{N}=\eta_{e}(u)
$$


and

$$
\begin{aligned}
& \xi_{e}(u)=-\frac{N}{2 u} \frac{\sum_{l=0}^{N / 2}(-1)^{l}\left(\begin{array}{l}
N \\
2 l
\end{array}\right) \sum_{k=0}^{l}(-1)^{k}\left(\begin{array}{l}
l \\
k
\end{array}\right) u^{l-k}}{\sum_{l=0}^{N / 2-1}(-1)^{l}\left(\begin{array}{c}
N \\
2 l+1
\end{array}\right) \sum_{k=0}^{l}(-1)^{k}\left(\begin{array}{l}
l \\
k
\end{array}\right) u^{l-k}}, \\
& \eta_{e}(u)=\frac{u^{N / 2}}{2 \sum_{l=0}^{N / 2-1}(-1)^{l}\left(\begin{array}{c}
N \\
2 l+1
\end{array}\right) \sum_{k=0}^{l}(-1)^{k}\left(\begin{array}{l}
l \\
k
\end{array}\right) u^{l-k}} .
\end{aligned}
$$

The highest order of polynomials involved in the above equations is $N / 2-1$. The solution for $\tilde{R}_{N}$ looks similar to the previous ones in equation (13), (A.4):

$$
\begin{aligned}
\tilde{R}_{N}(u) & =\frac{1}{\mu(u)} \int \eta_{e}(u) \mu(u) \mathrm{d} u, \\
\mu(u) & =\exp \left[\int \xi_{e}(u) \mathrm{d} u\right] .
\end{aligned}
$$

\section{A.2.1 Square}

Substituting $N=4$ in equations (A.10) gives the following expressions for the solution (A.11):

$$
\begin{aligned}
\mu(u) & =\frac{\mathrm{e}^{u / 2}}{u^{2}(u-2)}, \\
\tilde{R}_{4}(u) & =\frac{1}{8} u^{2}\left[1+\left(1-\frac{u}{2}\right) \mathrm{e}^{1-u / 2} E i\left(\frac{u}{2}-1\right)\right],
\end{aligned}
$$

where $\operatorname{Ei}(x)$ is the exponential integral function [22]. Passing to $r$ in this equation via the transformation $u \rightarrow(2 r / a)^{2}$ gives for the gauge transformed vector potential (14):

$$
\begin{aligned}
\tilde{A}_{r}= & -\frac{1}{4 \sqrt{2}} H a(1+z)^{3 / 2} \\
& \times\left[-1+z+\left(1+2 z-z^{2}\right) \mathrm{e}^{-z} \operatorname{Ei}(z)\right] \sin 4 \varphi \\
\tilde{A}_{\varphi}= & \frac{1}{2} H r+\frac{1}{2 \sqrt{2}} H a(1+z)^{3 / 2}\left[1-z \mathrm{e}^{-z} \operatorname{Ei}(z)\right] \cos 4 \varphi \\
z= & 2(r / a)^{2}-1
\end{aligned}
$$

\section{A.2.2 Regular hexagon}

In the case $N=6$ the polynomials in r.h.s. of equations (A.10) are of the third order. The solution for $\tilde{R}_{6}$ is obtained in the form of one single integral:

$$
\begin{aligned}
\mu(u) & =\frac{\mathrm{e}^{u / 2}}{u^{3}(u-4)^{3}(3 u-4)^{1 / 3}}, \\
\tilde{R}_{6}(u) & =\frac{1}{2 \mu(u)} \int \frac{\mu(u) u^{3}}{32-32 u+6 u^{2}} \mathrm{~d} u .
\end{aligned}
$$

\section{References}

1. V.V. Moshchalkov, L. Gielen, C. Strunk, R. Jonckheere, X. Qiu, C. Van Haesendonck, Y. Bruynseraede, Nature (London) 373, 319 (1995)

2. P.-G. de Gennes, Superconductivity of Metals and Alloys (Benjamin, New York, 1966)

3. V.M. Fomin, V.R. Misko, J.T. Devreese, V.V. Moshchalkov, Phys. Rev. B 58, 11703 (1998)

4. V.A. Schweigert, F.M. Peeters, Phys. Rev. B 60, 3084 (1999)

5. D. Saint-James, G. Sarma, E.J. Thomas, Type II Superconductivity (Pergamon Press, Oxford, 1973)

6. A. Houghton, F.B. McLean, Phys. Lett. 19, 172 (1965)

7. V.M. Fomin, J.T. Devreese, V.V. Moshchalkov, Europhys. Lett. 42, 553 (1998)

8. D. Saint-James, Phys. Lett. 15, 13 (1965)

9. H.J. Fink, A.G. Presson, Phys. Rev. 151, 219 (1966)

10. V.V. Moshchalkov, X.G. Qiu, V. Bruyndoncx, Phys. Rev. B 55, 11793 (1997)

11. P.S. Deo, V.A. Schweigert, F.M. Peeters, A.K. Geim, Phys. Rev. Lett. 79, 4653 (1997)

12. V.A. Schweigert, F.M. Peeters, Phys. Rev. Lett. 83, 2409 (1999)

13. L.F. Chibotaru, A. Ceulemans, V. Bruyndoncx, V.V. Moshchalkov, Nature 408, 833 (2000)

14. L.F. Chibotaru, A. Ceulemans, V. Bruyndoncx, V.V. Moshchalkov, Phys. Rev. Lett. 86, 1323 (2001)

15. V. Bruyndoncx, J.G. Rodrigo, T. Puig, L. Van Look, V.V. Moshchalkov, Phys. Rev. B 60, 4285 (1999)

16. L.D. Landau, E.M. Lifshitz, Theory of Fields (Mir, Moscow, 1970)

17. G.A. Korn, T.M. Korn, Mathematical Handbook for Scientists and Engineers (McGraw-Hill, New York, 1968)

18. L.D. Landau, E.M. Lifshitz, Quantum Mechanics, 2nd edn. (Pergamon, Oxford, 1975)

19. Vorticity (winding number) is an integer, $L$, measuring the total change of the phase of the order parameter, $2 \pi L$, along a closed boundary line of the sample. The total winding number is composed of separate, spatially resolved circulations of the gradient of phase of the order parameter corresponding to winding numbers $L_{i}$, so that $L=\sum_{i} L_{i}$. Regions of positive circulation with respect to the direction of the applied field, $L_{i}>0$, are called vortices (they induce local paramagnetic currents); in the opposite case, $L_{i}<0$, they are called antivortices. In bulk superconductors each vortex contributes by $L_{i}$ flux quanta $\left(\Phi_{0}\right)$ to the total quantised flux $\left(L \Phi_{0}\right)$ which threads the sample. In the case $L_{i}=1$ we have a normal (or $\Phi_{0^{-}}$) vortex and for $L_{i}=-1$ a normal (or $\Phi_{0^{-}}$) antivortex

20. L.F. Chibotaru, A. Ceulemans, G. Teniers, V.V. Moshchalkov, Physica C 369, 149 (2002)

21. V.V. Kabanov, J. Bonca, private communication; Phys. Rev. B 65, 012509 (2002)

22. Handbook of Mathematical Functions, edited by M. Abramowitz, I. Stegun (Dover, New York, 1970)

23. A. Barone, G. Paternò, Physics and Applications of the Josephson Effect (J. Wiley and Sons, New York, 1982)

24. E.H. Brandt, Physica C 332, 99 (2000) 